\title{
CENTER-BY-METABELIAN GROUPS OF PRIME EXPONENT BY
}

JAY I. MILLER

\begin{abstract}
We show that a center-by-metabelian group of prime exponent $p$ is nilpotent of class at most $p$, and this result is best possible. The proof is based on techniques dealing with varieties of groups.
\end{abstract}

1. Introduction. A classic result due to Meier-Wunderli [5] states that a metabelian group of prime exponent $p$ is nilpotent of class at most $p$. Thus a center-by-metabelian group of exponent $p$ is nilpotent of class at most $p+1$. We show below that this bound can be reduced to $p$. Because such groups do exist for every prime $p \geqslant 5[4$, Satz 6], the bound is best possible.

2. Notation and terminology. Our notation is generally the same as that in [6], to which we also refer the reader for elementary results concerning varieties of groups. We, however, use capital (small) italic letters for groups (elements). The variety generated by a particular group $G$ we denote $\operatorname{var}(G)$, and $\{1\}$ denotes the trivial group.

Commutators are left-normed, with $\left(x_{1}, x_{2}, \ldots, x_{n}\right)$ an element in $G_{n}$, the $n$th term of the descending central series of $G$. The nth Engel law is the varietal law $(x, n y)$, defined inductively:

$$
(x, 0 y)=x, \quad(x, n y)=((x,(n-1) y), y) .
$$

We abbreviate $((x, y),(u, v))$ by $(x, y ; u, v)$ and $((x, y ; u, v), w)$ by $(x, y$; $u, v ; w)$. Thus $G$ is metabelian if it satisfies the law $(x, y ; u, v)$; and center-by-metabelian, if it satisfies the law $(x, y ; u, v ; w)$. We call $(x, y ; u, v)$ a double commutator.

$H$ is a factor group of $G$ if $H \cong G / N$ for some $N<G$. $A$ is a factor of $G$ if $A \simeq H / K$, where $\{1\} \leqslant K<H \leqslant G$. A finite group is critical if it is not contained in the variety generated by its proper factors. A group is basic if it is critical and generates a join-irreducible variety. The $p$-group $G$ is regular if for every $a, b \in G,(a, b)^{p}=a^{p} b^{p} c^{p}$, where $c$ is a commutator word in $a$ and $b$. A variety is regular if every finite group in it is regular.

Received by the editors July 8, 1977.

AMS (MOS) subject classifications (1970). Primary 20D10, 20D15, 20E10, 20F45; Secondary $20 \mathrm{E} 15$.

Key words and phrases. Basic, basis, center-by-metabelian, Engel law, law, regular, relatively free, variety. 
3. The main result. Let $H$ be a center-by-metabelian group of prime exponent $p$. Then $\operatorname{var}(H)$ has exponent $p$, it is regular and it is center-bymetabelian. To show that $H$ is nilpotent of class at most $p$, we first show that every basic group in $\operatorname{var}(H)$ has that property. From here on, therefore, let $G$ denote a basic center-by-metabelian group of exponent $p, p \geqslant 5$, and assume that $G$ is nilpotent of the maximum possible class, $p+1$. Also, let $F$ be a finitely generated relatively free group that generates $\operatorname{var}(G)$.

LEMMA 3.1. In $\operatorname{var}(G), 2$-generator groups have class at most p. Equivalently, 2-variable words of weight $p+1$ are trivial.

Proof. We first note that $F^{\prime \prime} \leqslant Z(F)$ because $\operatorname{var}(G)$ is center-by-metabelian. $F / F^{\prime \prime}$ is a finite metabelian $p$-group such that every finite group in $\operatorname{var}\left(F / F^{\prime \prime}\right)$ is regular. By [8, Theorem 1.4], every 2-generator subgroup of $F / F^{\prime \prime}$ has class less than $p$. In $F$, therefore, every commutator of weight $p$ in 2 variables is contained in $F^{\prime \prime}$. Since $F^{\prime \prime} \leqslant Z(F)$, every commutator of weight $p+1$ in 2 variables is trivial. Q.E.D.

Proposition 3.2. $\operatorname{var}(G)$ satisfies the $(p-1)$ th Engel law.

Proof. Let $x$ and $y$ be elements of a free set of generators of $F$. Then $(x,(p-1) y) \in F_{p+1}$ because $F$ has exponent $p[3,18.4 .13]$. Thus in $F$, $(x,(p-1) y)$ can be written as a product of simple commutators in $x$ and $y$, each of weight $p+1$. By the lemma, each commutator in this product is trivial. Therefore $F$ and, hence, $\operatorname{var}(G)$ satisfy the law $(x,(p-1) y)=1$. Q.E.D.

Proposition 3.3. Let $a, b$ and $y$ be elements of $a$ free set $S$ of generators of $F$. Then in $F$, every simple commutator of the form $(a, r y, b, s y)$ is trivial, where $r+s=p-1$.

Proof. Since $F$ satisfies the $(p-1)$ th Engel law, both $(a, b,(p-1) y)$ and $(a,(p-1) y, b)$ are trivial.

As a metabelian group, $F / F^{\prime \prime}$ satisfies the Witt identity. Thus

$$
(a, b, y)(b, y, a)(y, a, b) \in F^{\prime \prime} .
$$

Since $F^{\prime \prime}<Z(F)$, we get

$$
((a, b, y)(b, y, a)(y, a, b),(p-2) y)=1 .
$$

Now

$$
((a, b, y)(b, y, a)(y, a, b), y)=(a, b, y, y)(b, y, a, y)(y, a, b, y) g_{7},
$$

where $g_{7} \in F_{7}$. 
Proceeding by induction, we obtain

$$
\begin{aligned}
1 & =((a, b, y)(b, y, a)(y, a, b),(p-2) y) \\
& =(a, b, y,(p-2) y)(b, y, a,(p-2) y)(y, a, b,(p-2) y) g_{p+4},
\end{aligned}
$$

where $g_{p+4} \in F_{p+4}$. Since $F_{p+4}=\{1\}$ and $(a, b,(p-1) y)=1$, we obtain the following equation:

$$
(b, y, a,(p-2) y)=(y, a, b,(p-2) y)^{-1}=(a, y, b,(p-2) y) .
$$

Since 2-variable commutators of weight $p+1$ are trivial in $F$,

$$
(x, y, x,(p-2) y)=1,
$$

where $x \in S$. Replacing $x$ by $a b$ and expanding, we get

$$
(a, y, b,(p-2) y)(b, y, a,(p-a) y)=1 .
$$

Equation (A) then implies that $(a, y, b,(p-2) y)^{2}=1$. Since $p>2$, we have $(a, y, b,(p-2) y)=1$.

Thus if $r=0,1$ or $p-1,(a, r y, b, s y)=1$ in $F$, where $r+s=p-1$. Continuing by induction, assume $1 \leqslant r<p-2$ and $(a, r y, b, s y)=1$. Now

$$
((a, r y), y, b)(y, b,(a, r y))(b,(a, r y), y) \in F^{\prime \prime}
$$

by the Witt identity, and since $F^{\prime \prime} \leqslant Z(F)$,

$$
(((a, r y) y, b)(y, b,(a, r y))(b,(a, r y), y),(s-1) y)=1 .
$$

Reasoning as above, and expanding, this yields

$$
(a, r y, y, b,(s-1) y)(y, b,(a, r y),(s-1) y)(b,(a, r y), y,(s-1) y)=1 .
$$

Since $(y, b,(a, r y)) \in F^{\prime \prime} \leqslant Z(F),(y, b,(a, r y),(s-1) y)=1$. Also

$$
(b,(a, r y), y,(s-1) y)=((a, r y), b, y,(s-1) y)^{-1}=(a, r y, b, s y)^{-1},
$$

which is trivial by the induction hypothesis. Thus (B) becomes

$$
(a,(r+1) y, b,(s-1) y)=1,
$$

and this induction step completes the proof. Q.E.D.

COROLlary 3.4. In $F,(a, r y ; b, s y)=1$, where $r$ and $s$ are positive integers such that $r+s=p-1$. Hence $(a, r y ; b$, sy) is a law in $\operatorname{var}(G)$.

Proof. Since $(a, r y ; b, s y)$ is a double commutator of weight $p+1$, it can be written as a product of simple left-normed commutators of weight $p+1$, each of which contains exactly one $a$, one $b$, and $p-1 y$ 's. By the proposition, each of these is trivial. Q.E.D.

Lemma 3.5. Let $\left\{a, b, x, y, y_{1}, y_{2}, \ldots, y_{p-3}\right\}$ be a free set of generators of $F$. Then the following laws hold in $F$ : 


$$
\left(a, b ; x, y, y_{1}, y_{2}, \ldots, y_{p-3}\right)=\left(a, b ; x, y, y_{\sigma(1)}, y_{\sigma(2)}, \ldots, y_{\sigma(p-3)}\right),
$$

where $\sigma$ is any permutation of $\{1,2, \ldots, p-3\}$.

$$
\begin{gathered}
\left(a, b ; x, y, y_{1}, \ldots, y_{p-3}\right)^{-1}=\left(b, a ; x, y, y_{1}, \ldots, y_{p-3}\right) \\
=\left(a, b ; y, x, y_{1}, \ldots, y_{p-3}\right)=\left(a^{-1}, b ; x, y, y_{1}, \ldots, y_{p-3}\right) \\
=\cdots=\left(a, b ; x, y, y_{1}, \ldots, y_{p-3}^{-1}\right)=\left(x, y, y_{1}, \ldots, y_{p-3} ; a, b\right) . \\
\left(a, b ; x, y, y_{1}, y_{2}, \ldots, y_{p-3}\right)\left(a, b ; y, y_{1}, x, y_{2}, \ldots, y_{p-3}\right) \\
\quad \times\left(a, b ; y_{1}, x, y, y_{2}, \ldots, y_{p-3}\right)=1 . \\
\text { Let } d_{1}, d_{2} \in F^{\prime} . \text { Then }\left(d_{1}, a, d_{2}\right)=\left(d_{1} ; d_{2}, a^{-1}\right) .
\end{gathered}
$$

Proof. The following are well-known properties of metabelian groups:

(i) $\left(x, y, y_{1}, y_{2}, \ldots, y_{p-3}\right)=\left(x, y, y_{\sigma(1)}, y_{\sigma(2)}, \ldots, y_{\sigma(p-3)}\right)$, where $\sigma$ is any permutation of $\{1,2, \ldots, p-3\}$.

(ii) $(a, b)^{-1}=(b, a)$.

(iii) $\left(x, y, y_{1}\right)\left(y, y_{1}, x\right)\left(y_{1}, x, y\right)=1$ (the Witt identity).

With these, parts (1), (2) and (3) of the lemma follow easily since $F$ is center-by-metabelian, and all double commutators are of maximal weight. Part (4) is Lemma 6.2 of [2]. Q.E.D.

Proposition 3.6. In $F$, commutators of weight 2 commute with commutators of weight $p-1$; i.e., $\left(F_{2}, F_{p-1}\right)=1$.

Proof. Let $\left\{a, b, x, y, y_{1}, y_{2}, \ldots, y_{p-2}\right\}$ be a free set of generators of $F$. It suffices to show that $\left(a, b ; x, y_{1}, y_{2}, \ldots, y_{p-2}\right)$ is a law in $F$.

When working with laws in $F$, we shall use the notation " $a \mapsto b$ " to mean that each occurrence of $a$ is replaced by $b$. Then " $a \leftrightarrow b$ " will mean $a \mapsto b$ and $b \mapsto a$ simultaneously.

Also, we shall use the term "separation" to denote the following procedure: Assume $1=f_{1} f_{2} \cdots f_{r}$ is a law in $F$, where $r<p$ and each $f_{i}$ is a product of nontrivial commutators of maximal weight, each one containing exactly $i$ occurrences of $x$. Then each $f_{i}$ is a law in $F, i=1,2, \ldots, r$. (See, e.g., [1, Corollary 1.1] for a more detailed description of this.)

We now proceed to the proof of the proposition. In the law

$$
1=(a, y ; b,(p-2) y),
$$

which is valid by (3.4), let $y \mapsto y y_{1}$. After some expanding, we get

$$
\begin{aligned}
1= & \left(a, y ; b, y,(p-3) y y_{1}\right)\left(a, y_{1} ; b, y,(p-3) y y_{1}\right) \\
& \times\left(a, y ; b, y_{1},(p-3) y y_{1}\right)\left(a, y_{1} ; b, y_{1},(p-3) y y_{1}\right) .
\end{aligned}
$$

Using separation, we obtain from this a law written as the product of double commutators, in which exactly one $y_{1}$ occurs in each double commutator. We 
thus have

$1=\left(a, y ; b, y, y_{1},(p-4) y\right)^{p-3}\left(a, y_{1} ; b, y,(p-3) y\right)\left(a, y ; b, y_{1},(p-3) y\right)$.

Since $F$ has exponent $p$, this becomes

$$
1=\left(a, y ; y, b, y_{1},(p-4) y\right)^{3}\left(a, y_{1} ; b,(p-2) y\right)\left(a, y ; b, y_{1}, y,(p-4) y\right) \text {. }
$$

Using 3.5(3) and 3.5(2), we get that

$$
\begin{aligned}
\left(a, y ; b, y_{1}, y,(p-4) y\right) & \\
\quad= & \left(a, y ; y, y_{1}, b,(p-4) y\right)\left(a, y ; b, y, y_{1},(p-4) y\right) .
\end{aligned}
$$

Inserting this in equation $(\mathrm{A})$ yields

$$
\begin{aligned}
1= & \left(a, y ; y, b, y_{1},(p-4) y\right)^{3}\left(a, y_{1} ; b,(p-2) y\right) \\
& \times\left(a, y ; y, y_{1}, b,(p-4) y\right)\left(a, y ; b, y, y_{1},(p-4) y\right) \\
= & \left(a, y ; y, b, y_{1},(p-4) y\right)^{2}\left(a, y_{1} ; b,(p-2) y\right)\left(a, y ; y, y_{1}, b,(p-4) y\right) \\
= & \left(a, y ; y, b,(p-4) y, y_{1}\right)^{2}\left(a, y_{1} ; b,(p-3) y, y\right) \\
& \times\left(a, y ; y, y_{1}, b,(p-4) y\right) \\
= & \left(a, y, y_{1}^{-1} ; y, b,(p-4) y\right)^{2}\left(a, y_{1}, y^{-1} ; b,(p-3) y\right) \\
& \times\left(a, y ; y, y_{1}, b,(p-4) y\right) .
\end{aligned}
$$

Using 3.5(2) we rewrite this as

$1=\left(a, y, y_{1} ; b, y,(p-4) y\right)^{2}\left(y_{1}, a, y ; b,(p-3) y\right)\left(a, y ; y, y_{1}, b,(p-4) y\right)$.

Using the Witt identity, we have

$$
\left(y_{1}, a, y ; b,(p-3) y\right)=\left(y, a, y_{1} ; b,(p-3) y\right)\left(y_{1}, y, a ; b,(p-3) y\right) \text {, }
$$

from which equation (B) becomes

$$
1=\left(a, y, y_{1} ; b,(p-3) y\right)\left(y_{1}, y, a ; b,(p-3) y\right)\left(a, y ; y, y_{1}, b,(p-4) y\right) \text {. }
$$

Taking inverses and rearranging yields

$$
1=\left(a, y ; y_{1}, y, b,(p-4) y\right)\left(y, a, y_{1} ; b,(p-3) y\right)\left(y, y_{1}, a ; b,(p-3) y\right) \text {. }
$$

Now from (3.4), $1=(a, y ; b,(p-3) y)$. Letting $y \mapsto x y$, we get, after some expanding,

$$
\begin{aligned}
1= & (a, x ; b, x,(p-3) x y)(a, y ; b, y,(p-3) x y) \\
& \times(a, x ; b, y,(p-3) x y)(a, y ; b, x,(p-3) x y) .
\end{aligned}
$$


Using separation, we obtain from this a law in which each double commutator contains exactly one $x$. We get

$$
\begin{aligned}
1 & =(a, y ; b, y, x,(p-4) y)^{p-3}(a, x ; b, y,(p-3) y)(a, y ; b, x,(p-3) y) \\
& =(a, y ; b, y,(p-4) y, x)^{-3}(a, x ; b,(p-3) y, y)(a, y ; b, x,(p-3) y) \\
& =\left(a, y, x^{-1} ; b,(p-3) y\right)^{-3}\left(a, x, y^{-1} ; b,(p-3) y\right)(a, y ; b, x,(p-3) y),
\end{aligned}
$$

or

$$
1=(a, y, x ; b,(p-3) y)^{3}(x, a, y ; b,(p-3) y)(a, y ; b, x,(p-3) y) \text {. }
$$

Using the Witt identity again, we have

$$
(x, a, y ; b,(p-3) y)=(y, a, x ; b,(p-3) y)(x, y, a ; b,(p-3) y) .
$$

Substituting this into (D) yields

$$
1=(a, y, x ; b,(p-3) y)^{2}(x, y, a ; b,(p-3) y)(a, y ; b, x,(p-3) y) .
$$

Letting $x \leftrightarrow b$, we get

$$
1=(a, y, b ; x,(p-3) y)^{2}(b, y, a ; x,(p-3) y)(a, y ; x, b,(p-3) y) .
$$

Multiplying these last two laws yields

$$
\begin{aligned}
1= & (a, y, x ; b,(p-3) y)^{2}(a, y, b ; x,(p-3) y)^{2} \\
& \times(x, y, a ; b,(p-3) y)(b, y, a ; x,(p-3) y) .
\end{aligned}
$$

We now note the following:

$$
\begin{aligned}
(b, y, a ; x,(p-3) y) & =\left(b, y ; x,(p-3) y, a^{-1}\right) \\
& =\left(b, y ; x, y, a^{-1},(p-4) y\right) \\
& =\left(b, y, y^{-1} ; x, y, a^{-1},(p-5) y\right)=\cdots \\
& =\left(b, y,(p-4) y^{-1} ; x, y, a^{-1}\right) \\
& =\left(b, y,(p-4) y ; x, y, a^{-1}\right)^{(-1)^{p-4}} \\
& =(b,(p-3) y ; x, y, a) .
\end{aligned}
$$

Using this in equation (E) yields

$$
1=[(a, y, x ; b,(p-3) y)(a, y, b ; x,(p-3) y)]^{2} .
$$

Because $p$ is odd, we get

$$
1=(a, y, x ; b,(p-3) y)(a, y, b ; x,(p-3) y) .
$$


From equation (C), with $a \leftrightarrow b$ and $y_{1} \mapsto x$, we obtain

$$
\begin{aligned}
1 & =(b, y ; x, y, a,(p-4) y)(y, b, x ; a,(p-3) y)(y, x, b ; a(p-3) y) \\
& =\left(b, y, a^{-1} ; x,(p-3) y\right)(y, b, x ; a,(p-3) y)(y, x, b ; a,(p-3) y) .
\end{aligned}
$$

Taking inverses yields

$$
1=(b, y, a ; x,(p-3) y)(b, y, x ; a,(p-3) y)(x, y, b ; a,(p-3) y) .
$$

Now from (I), with $a \leftrightarrow b$, we get

$$
1=(b, y, x ; a,(p-3) y)(b, y, a ; x,(p-3) y) .
$$

Using this, we eliminate the first two factors in $(\mathrm{J})$, getting

$$
1=(x, y, b ; a,(p-3) y) \text {. }
$$

Now using the Witt identity one last time, we have

$$
1=(x, y, b ; a,(p-3) y)(y, b, x ;(p-3) y)(b, x, y ; a,(p-3) y) .
$$

Therefore, by using $(\mathrm{K})$ and then taking inverses, we get

$$
1=(b, y, x ; a,(p-3) y)(x, b, y ; a,(p-3) y) .
$$

From this, and from (K) with $b \leftrightarrow x$, we obtain

$$
1=(x, b, y ; a,(p-3) y)=\left(x, b ; a,(p-3) y, y^{-1}\right) .
$$

Letting $a \leftrightarrow x$ and taking inverses yields

$$
1=(a, b ; x,(p-2) y) .
$$

Thus, $F / Z\left(F^{\prime}\right)$ satisfies the law $(x,(p-2) y)$. Since $F^{\prime \prime} \leqslant Z\left(F^{\prime}\right)$ and $F_{p} \leqslant Z\left(F^{\prime}\right), F / Z\left(F^{\prime}\right)$ is a metabelian group of class at most $p-1$. By a well-known result [1, Corollary 2.1], any metabelian group of small class satisfying the law $(x,(p-2) y)$ also satisfies the law $\left(x, y_{1}, y_{2}, \ldots, y_{p-2}\right)$. Therefore $F / Z\left(F^{\prime}\right)$ satisfies this law, and $\left(a, b ; x, y_{1}, y_{2}, \ldots, y_{p-2}\right)$ is a law in $F$. Q.E.D.

THEOREM 3.7. Let $G$ be a basic center-by-metabelian group of prime exponent $p$. Then $G$ is nilpotent of class at most $p$.

Proof. Let $F$ be a relatively free group that generates $\operatorname{var}(G)$, having $\left\{a, b, x, y_{1}, y_{2}, \ldots, y_{p-2}\right\}$ as a free set of generators. It is known that $F$ is nilpotent of class at most $p+1$; so assume $F$ has class exactly $p+1$. From the above proposition, we have that $1=\left(a, b ; x, y_{1}, y_{2}, \ldots, y_{p-2}\right)$. By 3.5(4), we get $1=\left(a, b, y_{p-2}^{-1} ; x, y_{1}, y_{2}, \ldots, y_{p-3}\right)$, and taking inverses yields $1=$ $\left(a, b, y_{p-2} ; x, y_{1}, y_{2}, \ldots, y_{p-3}\right)$.

Proceeding by induction, we see that every double commutator of weight $p+1$ is trivial. Because $F / F^{\prime \prime}$ is a metabelian group of exponent $p, F_{p+1} \leqslant$ $F^{\prime \prime}[5]$. Thus the simple commutator $\left(a, b, x, y_{1}, y_{2}, \ldots, y_{p-2}\right)$ can be written as a product of double commutators, each of weight $p+1$ and containing all 
the generators of $F$. Since these are all trivial, so is $F_{p+1}$. Therefore, $F$ has class at most $p$; and, hence, so does $G$. Q.E.D.

THEOREM 3.8. Let $H$ be a center-by-metabelian group of prime exponent $p$. Then $H$ is nilpotent of class at most $p$.

Proof. Since groups of exponent 2 are abelian, and groups of exponent 3 are nilpotent of class at most 3 [6, Theorem 34.32], $H$ is metabelian if $p<5$. Thus we may assume that $p \geqslant 5$ and $H$ is not metabelian.

Since $H$ is center-by-metabelian but not metabelian, $Z(H)>\{1\}$ and $H^{\prime \prime} \leqslant Z(H)$. Therefore $H$ and, hence, $\operatorname{var}(H)$ are solvable. Because a solvable group of exponent $p$ is locally finite [7, Theorem 7.16], $\operatorname{var}(H)$ is a locally finite variety. Hence $\operatorname{var}(H)$ is generated by its basic groups, and it remains only to show that those are nilpotent of class at most $p$.

Let $G$ be a basic group in $\operatorname{var}(H)$. If $G$ is abelian, it has class 1 ; and if $G$ is metabelian, it has class at most $p$ since every metabelian group of exponent $p$ does.

If $G$ is not metabelian, it must be a basic center-by-metabelian group of exponent $p$. Hence it has class at most $p$ by 3.7. Therefore all the basic groups in $\operatorname{var}(H)$ have class at most $p$. Hence $H$ itself is nilpotent of class at most $p$. Q.E.D.

4. Acknowledgments. The major results in this paper are taken from the author's doctoral dissertation, written under Professor Paul M. Weichsel at the University of Illinois. The author would also like to acknowledge an important observation regarding Theorem 3.8 pointed out by Professor Derek J. S. Robinson.

\section{BIBLIOGRAPHY}

1. W. Brisley, On varieties of metabelian p-groups, and their laws, J. Austral. Math. Soc. 7 (1967), 64-80.

2. C. K. Gupta, A faithful matrix representation for certain centre-by-metabelian groups, J. Austral. Math. Soc. 10 (1969), 451-454.

3. M. Hall, Jr., The theory of groups, Macmillan, New York, 1959.

4. H. Meier-Wunderli, Uber endliche p-Gruppen, deren Elementen der Gleichung $x^{p}=1$ genügen, Comment. Math. Helv. 24 (1950), 18-45.

5. Metabelsche Gruppen, Comment. Math. Helv. 25 (1951), 1-10.

6. H. Neumann, Varieties of groups, Springer-Verlag, New York, 1967.

7. D. J. S. Robinson, Finiteness conditions and generalized soluble groups, Part 2, SpringerVerlag, New York, 1972.

8. P. M. Weichsel, Regular p-groups and varieties, Math. Z. 95 (1967), 223-231.

Department of Mathematics, Marquette University, Milwaukee, Wisconsin 53233

Current address: 8454 N. 57th Street, Brown Deer, Wisconsin 53223 